УДК 531/534: [57+61]

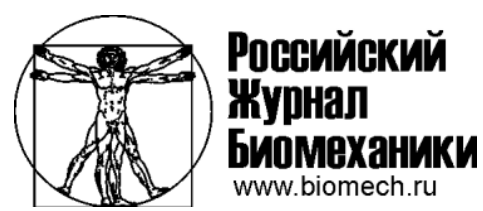

\title{
ОБЛАСТИ СОПРОТИВЛЕНИЯ ДЛЯ МОДЕЛИ ОДНОКОРЕННОГО ЗУБА: РАЗЛИЧНЫЕ СЛУЧАИ СИММЕТРИИ
}

\author{
С.М. Босяков, А.Ф. Мселати
}

Кафедра теоретической и прикладной механики Белорусского государственного университета, Беларусь, 220030, Минск, проспект Независимости, 4, e-mail: bosiakov@bsu.by

\begin{abstract}
Аннотация. В работе представлены результаты нахождения областей сопротивления и положения прямых поступательного воздействия для корня зуба в форме составного параболоида на основании подхода, предложенного А.Л. Дубининым, Ю.И. Няшиным и М.А. Осипенко. Жесткость периодонтальной связки получена в результате решения задачи о равновесии упруго закрепленного твердого тела при действии сосредоточенной нагрузки. Периодонтальная связка является линейно упругой и почти несжимаемой; ее толщина постоянна по нормали к внешней поверхности. Использовано представление для малых перемещений корня зуба через комбинацию поступательных перемещений и углов поворота. Предполагается, что полная деформация тканей периодонта по нормали к поверхности корня совпадает с шириной периодонтальной щели в этом направлении. Внешняя поверхность корня зуба и периодонтальной связки описываются уравнениями параболоидов. Выведены уравнения для областей сопротивления асимметричного корня зуба, корня зуба с одной плоскостью симметрии, симметричного корня зуба с эллиптическим и круговым поперечными сечениями. Показано, что для асимметричного корня зуба областью сопротивления является горловой эллипс однополостного гиперболоида; для корня зуба с одной плоскостью симметрии и симметричного корня зуба с эллиптическим поперечным сечением область сопротивления - две точки; для симметричного корня зуба с круговым сечением существует единый центр сопротивления. Проведен расчет положений областей сопротивления для асимметричных и симметричных корней зубов с различными геометрическими размерами. Выполнена визуализация областей сопротивления, а также прямых поступательного воздействия для корня зуба с одной плоскостью симметрии и асимметричного корня зуба. Полученные результаты могут быть использованы для прогнозирования областей приложения и направления нагрузки для поступательного перемещения корня зуба.
\end{abstract}

Ключевые слова: область сопротивления, асимметричный корень зуба, периодонтальная связка, параболоид, прямые поступательного воздействия.

\section{ВведЕНИЕ}

Центр сопротивления рассматривается как наиболее важный ориентир при планировании краткосрочных и долгосрочных перемещений зубов. Это обусловлено тем, что сила, линия действия которой проходит через эту точку, приводит к поступательному движению зуба. Другие виды движения зубов, как правило, также описываются с учетом расположения центра сопротивления или кривых момент / сила $[3,4,8,10,14,15,17]$. 
История развития понятия «центр сопротивления», а также различные подходы к его определению представлены в фундаментальной работе [7]. Один из выводов авторов указывает на необходимость пересмотра этого понятия с учетом результатов последних исследований. К таким исследованиям, в частности, можно отнести работы $[18,20]$, посвященные численным экспериментам для асимметричной периодонтальной связки верхнечелюстного первого моляра. Результаты этих работ показали, что не существует единого центра сопротивления (центр сопротивления не является точкой в трехмерном пространстве) применительно к неидеализированному (асимметричному) корню зуба и периодонтальной связке. При планировании движения любого зуба предложено использовать трехмерные оси сопротивления, которые не пересекаются в одной точке [20]. Для клинических целей предлагается использовать малый трехмерный объем, ограниченный осями сопротивления, как объем, содержащий центры сопротивления, соответствующие поступательному перемещению зуба в различных направлениях. Аналогичный подход, основанный на использовании понятия радиуса сопротивления, предложен в работе [5]. Более строгий подход к определению условий поступательного перемещения корня зуба в периодонтальной связке, основанный на понятиях области сопротивления и прямых поступательного воздействия, предложен в работе [11]. Это определение получило развитие и обобщение в работе [6] для области сопротивления как конечного или бесконечного множества точек. В работах [6, 11] показано, что зуб получает поступательное перемещение (необязательно в направлении действия силы) в случае, если линия действия нагрузки проходит через точку области сопротивления и направлена соответствующим образом. Такая линия действия является прямой поступательного воздействия $[6,11]$. Также в работах $[6,11]$ показано, что область сопротивления может быть эллипсом (общий случай), двумя точками или одной точкой (исключительные случаи). Настоящее исследование развивает это актуальное направление для корней однокоренных зубов в форме составного параболоида. Целью работы является математическое моделирование области сопротивления для симметричных и асимметричных корней зубов.

\section{РАВНОВЕСИЕ КОРНЯ ЗУБА В ПЕРИОДОНТАЛЬНОЙ ОБОЛОЧКЕ}

При математическом моделировании напряженно-деформированного состояния системы «зуб - периодонтальная связка» в большинстве случаев корень зуба аппроксимируется абсолютно твердым телом в форме параболоида или гиперболоида $[2,12,16]$. Как показали результаты исследования [21], реальная форма однокоренного зуба с достаточно высокой точностью может быть аппроксимирована с помощью параболоида. Исходя из этого будем считать, что внешняя поверхность корня зуба (совпадающая с внутренней поверхностью периодонтальной связки) и внешняя поверхность периодонтальной связки, прилегающая к костной ткани зубной альвеолы (смещенная по нормали по отношению к поверхности корня зуба на величину $\delta$ ), описываются уравнениями параболоидов:

$$
\begin{gathered}
F_{k}(x, y, z)=y-h\left(\left(x / a_{k}\right)^{2}+\left(z / b_{k}\right)^{2}\right)=0, \\
F^{(k)}(x, y, z)=y+n_{y}^{(k)} \delta-h\left(\left(\frac{1}{a_{k}}\left(x+n_{x}^{(k)} \delta\right)\right)^{2}+\left(\frac{1}{b_{k}}\left(z+n_{z}^{(k)} \delta\right)\right)^{2}\right)=0,
\end{gathered}
$$


где $h$ - высота корня зуба; $a_{k}, b_{k}$ - полуоси эллипса в сечении корня зуба на уровне альвеолярного гребня, $a_{4}=a_{1}=\frac{b_{1}}{\sqrt{1-e_{t 1}^{2}}}, a_{3}=a_{2}=\frac{b_{2}}{\sqrt{1-e_{t 2}^{2}}}, b_{2}=b_{1}, b_{4}=b_{3}, e_{t 1}, e_{t 2}-$ эксцентриситеты эллипсов; $n_{x}^{(k)}, n_{y}^{(k)}, n_{z}^{(k)}$ - компоненты единичного вектора нормали к $k$-й поверхности корня зуба $(1), k=\overline{1,4}$; поверхности $F_{k}(x, y, z)$ и $F^{(k)}(x, y, z)$ при $k=1$ определены на промежутках $x \geq 0, y \geq 0$, при $k=2$ на $x<0, y \geq 0$, при $k=3$ на $x<0, y<0$ и при $k=4$ на $x \geq 0, y<0$.

При действии на зуб сосредоточенной силы $\vec{f}=\left(f_{x}, f_{y}, f_{z}\right)$ точки периодонта, прилегающие к поверхности корня зуба, получают перемещения, равные перемещению корня. Внешняя поверхность периодонтальной связки (2) является жестко закрепленной. Будем предполагать, что нагрузка сохраняется достаточно низкой, чтобы обеспечивать деформации периодонтальной связки, соответствующие начальному линейному участку диаграммы напряжения - деформации. В этом случае ткани периодонтальной связки с достаточно высокой точностью можно считать линейно упругим материалом $[9,19,20]$. Также в соответствии с работами $[12,13,16]$ будем считать периодонт несжимаемым материалом с коэффициентом Пуассона равным 0,49. С учетом этого компоненты тензора деформаций в системе координат, связанной с нормалью, образующей и направляющей к внешней поверхности корня зуба, можно определить следующим образом [12]:

$$
\varepsilon_{n n}=-u_{n} / \delta, \varepsilon_{t t}=\varepsilon_{\theta \theta}=0, \gamma_{n \theta}=-u_{\theta} / \delta, \gamma_{n t}=-u_{t} / \delta, \gamma_{t \theta}=0
$$

где $u_{n}, u_{t}, u_{\theta}-$ перемещения точек периодонта вдоль нормали, образующей и направляющей к поверхности корня зуба; $\delta$ - ширина периодонтальной связки в направлении нормали к поверхности (1). Нормаль $\vec{n}$, образующая $\vec{t}$ и направляющая $\vec{\theta}$ к поверхности корня зуба, а также его геометрические размеры показаны на рис. 1.

После преобразований уравнений равновесия корня зуба, описанных в работах $[1,16]$, получим систему однородных алгебраических уравнений относительно малых поступательных перемещений $\vec{u}_{0}=\left(u_{0 x}, u_{0 y}, u_{0 z}\right)$ и углов поворота корня зуба $\vec{\theta}=\left(\theta_{x}, \theta_{y}, \theta_{z}\right)$ следующего вида:

$$
\begin{gathered}
\left(\begin{array}{cc}
\mathrm{A} & \Gamma \\
\Gamma^{\mathrm{T}} & \mathrm{B}
\end{array}\right)\left(\begin{array}{c}
\vec{u}_{0} \\
\vec{\theta}
\end{array}\right)=\left(\begin{array}{l}
\vec{f} \\
\vec{m}
\end{array}\right), \\
\mathrm{A}=\left(\begin{array}{lll}
a_{11} & a_{12} & a_{13} \\
a_{21} & a_{22} & a_{23} \\
a_{31} & a_{32} & a_{33}
\end{array}\right), \mathrm{B}=\left(\begin{array}{lll}
a_{44} & a_{45} & a_{46} \\
a_{54} & a_{55} & a_{56} \\
a_{64} & a_{65} & a_{66}
\end{array}\right), \Gamma=\left(\begin{array}{lll}
a_{14} & a_{15} & a_{16} \\
a_{24} & a_{25} & a_{26} \\
a_{34} & a_{35} & a_{36}
\end{array}\right) .
\end{gathered}
$$

Здесь $a_{i j}=a_{j i}-$ коэффициенты, зависящие от физико-механических свойств и геометрических размеров периодонтальной связки; $\vec{m}=\left(m_{x}, m_{y}, m_{z}\right)=\vec{r} \times \vec{f}-$ главный момент внешних сил; $\vec{f}=\left(f_{x}, f_{y}, f_{z}\right)$ - главный вектор внешних сил; $\vec{r}=\left(x_{f}, y_{f}, z_{f}\right)-$ радиус-вектор; $x_{f}, y_{f}$ и $z_{f}$ - координаты точки приложения нагрузки. Выражения для коэффициентов $a_{i j}$ приведены в приложении. 


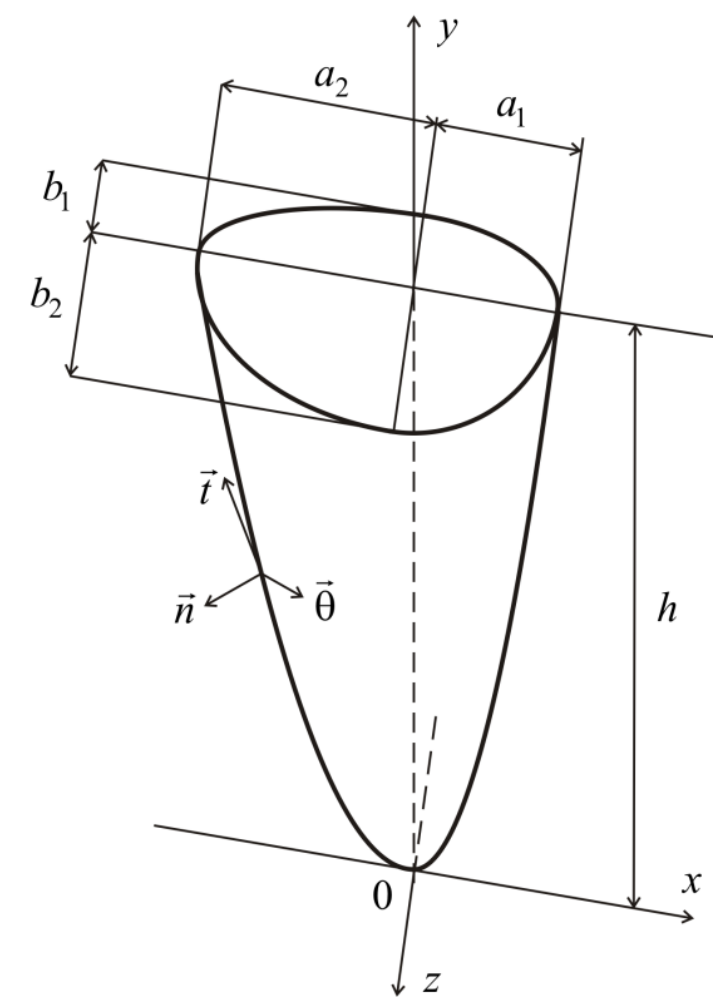

Рис. 1. Системы координат $(x, y, z)$ и $(\vec{n}, \vec{t}, \vec{\theta})$ и геометрические размеры асимметричного корня зуба, состоящего из четырех различных параболоидов

Решение системы (4) представим в матричном виде, предложенном в работе [11]:

$$
\left(\begin{array}{c}
\vec{u}_{0} \\
\vec{\theta}
\end{array}\right)=\left(\begin{array}{cc}
\alpha & \gamma \\
\gamma^{\mathrm{T}} & \beta
\end{array}\right)\left(\begin{array}{l}
\vec{f} \\
\vec{m}
\end{array}\right),
$$

где $\gamma^{\mathrm{T}}$ - транспонированная матрица $\gamma$.

\section{РАЗЛИЧНЫЕ СЛУЧАИ СИММЕТРИИ}

\section{Модель корня зуба в форме параболоида с круговым сечением}

Для корня зуба в форме кругового параболоида имеем $b=a_{1}=a_{2}=b_{1}=b_{3}$. С учетом ненулевых коэффициентов системы (4) матрицы системы (5) принимают вид

$$
\begin{gathered}
\alpha=\left(\begin{array}{ccc}
k_{11} & 0 & 0 \\
0 & k_{22} & 0 \\
0 & 0 & k_{11}
\end{array}\right), \beta=\left(\begin{array}{ccc}
k_{44} & 0 & 0 \\
0 & k_{55} & 0 \\
0 & 0 & k_{44}
\end{array}\right), \gamma=\left(\begin{array}{ccc}
0 & 0 & -k_{34} \\
0 & 0 & 0 \\
k_{34} & 0 & 0
\end{array}\right), \\
k_{11}=\frac{a_{44}}{a_{11} a_{44}-a_{34}^{2}}, k_{22}=\frac{1}{a_{22}}, k_{44}=\frac{a_{11}}{a_{11} a_{44}-a_{34}^{2}}, k_{55}=\frac{1}{a_{55}}, k_{34}=\frac{a_{34}}{a_{11} a_{44}-a_{34}^{2}} .
\end{gathered}
$$


Матрица $\delta$, являющаяся симметричной частью матрицы $\gamma \beta^{-1}$, представляет собой нулевую матрицу. Согласно $[6,11]$ в этом случае существует единый центр сопротивления корня зуба, координаты центра сопротивления определяются матрицей $\varepsilon$ (антисимметричной частью матрицы $\gamma \beta^{-1}$ ). Зуб принадлежит к типу $(0,0,0)$ по классификации [11]. Соответствующее преобразование матриц (6) показывает, что координаты центра сопротивления равны $\left(0,0, a_{34} / a_{11}\right)$. Любая сила, линия действия которой проходит через эту точку, приводит к поступательному перемещению зуба.

В табл. 1 приведены значения ненулевых коэффициентов $a_{i j}$. Радиус сечения корня на уровне альвеолярного гребня $b=3,9$ мм, высота корня $h=13,0$ мм; упругие свойства периодонтальной связки описываются константами $E=680$ кПа, $v=0,49$ [12, $15,16]$, толщина периодонтальной связки $\delta=0,229$ мм $[12,16]$.

C учетом данных табл. 1 координаты центра сопротивления равны $(0 ; 0 ; 8,431)$ мм.

\section{Модель корня зуба в форме параболоида с эллиптическим сечением}

В этом случае с коэффициентами $a_{i j}, i, j=\overline{1,6}$, при $a=a_{1}=a_{2}$ и $b=b_{1}=b_{2}$ получаем следующие матрицы:

$$
\begin{gathered}
\alpha=\left(\begin{array}{ccc}
k_{11} & 0 & 0 \\
0 & k_{22} & 0 \\
0 & 0 & k_{33}
\end{array}\right), \beta=\left(\begin{array}{ccc}
k_{44} & 0 & 0 \\
0 & k_{55} & 0 \\
0 & 0 & k_{66}
\end{array}\right), \gamma=\left(\begin{array}{ccc}
0 & 0 & k_{16} \\
0 & 0 & 0 \\
k_{34} & 0 & 0
\end{array}\right), \\
k_{11}=\frac{a_{66}}{a_{11} a_{66}-a_{16}^{2}}, k_{22}=\frac{1}{a_{22}}, k_{33}=\frac{a_{44}}{a_{33} a_{44}-a_{34}^{2}}, k_{44}=\frac{a_{33}}{a_{33} a_{44}-a_{34}^{2}}, \\
k_{55}=\frac{1}{a_{55}}, k_{66}=\frac{a_{11}}{a_{11} a_{66}-a_{16}^{2}}, k_{34}=\frac{a_{34}}{a_{33} a_{44}-a_{34}^{2}}, k_{16}=-\frac{a_{16}}{a_{11} a_{66}-a_{16}^{2}} .
\end{gathered}
$$

Матрица $\delta$ является ненулевой и имеет вид

$$
\delta=\left(\begin{array}{ccc}
0 & 0 & r_{13} \\
0 & 0 & 0 \\
r_{13} & 0 & 0
\end{array}\right), r_{13}=-\frac{a_{16} a_{33}+a_{11} a_{34}}{2 a_{11} a_{33}} .
$$

Собственные числа и векторы этой матрицы

$$
\delta_{1,2}=\mp r_{13}, \delta_{3}=0, \vec{v}_{1}=(1 / \sqrt{2}, 0,1 / \sqrt{2}), \vec{v}_{2}=(-1 / \sqrt{2}, 0,1 / \sqrt{2}), \vec{v}_{3}=(0,-1,0) \text {. }
$$

Таким образом, корень зуба в форме эллиптического параболоида принадлежит к типу $(+,-, 0)$ по классификации [11]. Прямые поступательного воздействия расположены в плоскостях $x=0$ и $z=0$. Область сопротивления образуют точки $P_{1}$ и $P_{2}$ с координатами $\left(0,0,-a_{16} / a_{11}\right)$ и $\left(0,0, a_{34} / a_{33}\right)$ соответственно. Если линия действия силы расположена в плоскости $x 0 y$ (или $z 0 y$ ) и проходит через точку $P_{1}$ (или $P_{2}$ ), то зуб получает поступательное перемещение в плоскости $x 0 y$ (или $z 0 y$ ). 
Таблиича 1

Коэффициенты $a_{i j}$ для модели корня зуба с круговым сечением

\begin{tabular}{|c|c|c|c|c|}
\hline$a_{11}=a_{33}, \mathrm{MH} / \mathrm{M}$ & $a_{22}, \mathrm{MH} / \mathrm{M}$ & $a_{44}=a_{66}, \mathrm{H}$ & $a_{55}, \mathrm{H}$ & $a_{16}=-a_{34}, \mathrm{\kappa H}$ \\
\hline 5,363 & 0,833 & 443,031 & 1,967 & $-45,214$ \\
\hline
\end{tabular}

Таблицุа 2

Коэффициенты $a_{i j}$ для модели корня зуба с эллиптическим сечением

\begin{tabular}{|c|c|c|c|c|c|c|c|}
\hline$a_{11}, \mathrm{MH} / \mathrm{M}$ & $a_{22}, \mathrm{MH} / \mathrm{M}$ & $a_{33}, \mathrm{MH} / \mathrm{M}$ & $a_{44}, \mathrm{H}$ & $a_{55}, \mathrm{H}$ & $a_{66}, \mathrm{H}$ & $a_{16}, \mathrm{\kappa H}$ & $a_{34}, \mathrm{\kappa H}$ \\
\hline 5,043 & 1,090 & 6,997 & 578,901 & 6,137 & 445,565 & $-44,168$ & 59,060 \\
\hline
\end{tabular}

Значения коэффициентов системы (4) приведены в табл. 2. Малая полуось эллипса на уровне альвеолярного гребня $b=3,9 \mathrm{мм}, a=\frac{b}{\sqrt{1-e_{t 1}^{2}}}, e_{t 1}=0,6$. Остальные геометрические размеры корня зуба и постоянные упругости периодонтальной связки имеют прежние значения.

В соответствии с данными таблицы координаты точек $P_{1}$ и $P_{2}$, образующих область сопротивления, равны $(0 ; 0 ; 8,759)$ мм и $(0 ; 0 ; 8,441)$ мм соответственно.

\section{Модель корня зуба в форме составного параболоида с одной плоскостью симметрии}

Для корня зуба, поверхность которого описывается двумя различными параболоидами, будем считать $b=b_{1}=b_{2}$. В этом случае корень зуба имеет одну плоскость симметрии $z=0$. Матрицы системы (6) записываются следующим образом:

$$
\begin{gathered}
\alpha=\left(\begin{array}{ccc}
k_{11} & k_{12} & 0 \\
k_{12} & k_{22} & 0 \\
0 & 0 & k_{33}
\end{array}\right), \beta=\left(\begin{array}{ccc}
k_{44} & k_{45} & 0 \\
k_{45} & k_{55} & 0 \\
0 & 0 & k_{66}
\end{array}\right), \gamma=\left(\begin{array}{ccc}
0 & 0 & k_{16} \\
0 & 0 & k_{26} \\
k_{34} & k_{35} & 0
\end{array}\right), \\
k_{11}=k_{\alpha}\left(a_{26}^{2}-a_{22} a_{66}\right), k_{12}=k_{\alpha}\left(a_{12} a_{66}-a_{16} a_{26}\right), k_{22}=k_{\alpha}\left(a_{16}^{2}-a_{11} a_{66}\right), \\
k_{33}=k_{\beta}\left(a_{45}^{2}-a_{44} a_{55}\right), k_{44}=k_{\beta}\left(a_{35}^{2}-a_{33} a_{55}\right), k_{55}=k_{\beta}\left(a_{34}^{2}-a_{33} a_{44}\right), \\
k_{45}=k_{\beta}\left(a_{33} a_{45}-a_{34} a_{35}\right), k_{66}=k_{\alpha}\left(a_{12}^{2}-a_{11} a_{22}\right), k_{16}=k_{\alpha}\left(a_{16} a_{22}-a_{12} a_{26}\right), \\
k_{26}=k_{\alpha}\left(a_{11} a_{26}-a_{12} a_{16}\right), k_{34}=k_{\beta}\left(a_{34} a_{55}-a_{35} a_{45}\right), k_{35}=k_{\beta}\left(a_{35} a_{44}-a_{34} a_{45}\right), \\
k_{\alpha}=a_{22}\left(a_{16}^{2}-a_{11} a_{66}\right)+a_{66} a_{12}^{2}-2 a_{12} a_{16} a_{26}+a_{11} a_{26}^{2}, \\
k_{\beta}=a_{44}\left(a_{35}^{2}-a_{33} a_{55}\right)+a_{34} a_{55}^{2}-2 a_{34} a_{35} a_{45}+a_{33} a_{45}^{2} .
\end{gathered}
$$


Матрица $\delta$ принимает вид

$$
\delta=\left(\begin{array}{ccc}
0 & 0 & r_{13} \\
0 & 0 & r_{23} \\
r_{13} & r_{23} & 0
\end{array}\right), r_{13}=\frac{1}{2}\left(\frac{a_{16} a_{22}-a_{12} a_{26}}{a_{12}^{2}-a_{11} a_{22}}-\frac{a_{34}}{a_{33}}\right), r_{23}=\frac{1}{2}\left(\frac{a_{11} a_{26}-a_{12} a_{16}}{a_{12}^{2}-a_{11} a_{22}}-\frac{a_{35}}{a_{33}}\right) \text {. }
$$

Собственные числа и векторы этой матрицы

$$
\delta_{1,2}=\mp \sqrt{r_{13}^{2}+r_{23}^{2}}, \delta_{3}=0, \vec{v}_{1,2}=\left(\frac{r_{13}}{\delta_{1,2}}, \frac{r_{23}}{\delta_{1,2}}, 1\right), \vec{v}_{3}=\left(-\frac{r_{23}}{r_{13}}, 1,0\right) .
$$

Отсюда следует, что корень зуба в форме составного параболоида с одной плоскостью симметрии, так же как и симметричный корень зуба с эллиптическим поперечным сечением, принадлежит к типу $(+,-, 0)$ по классификации [11].

Плоскости, в которых расположены прямые поступательного воздействия, определяются следующим уравнением:

$$
z\left(\left(y-y_{2}\right)\left(x-x_{3}\right)-\left(y-y_{1}\right)\left(x-x_{2}\right)\right)=0 .
$$

Областью сопротивления являются две точки: $P_{1}\left(x_{3}, 0, y_{1}\right)$ и $P_{2}\left(x_{2}, 0, y_{2}\right)$, $y_{1}=\frac{a_{16} a_{22}-a_{12} a_{26}}{a_{12}^{2}-a_{11} a_{22}}, \quad x_{2}=-\frac{a_{35}}{a_{33}}, \quad y_{2}=\frac{a_{34}}{a_{33}}, \quad x_{3}=\frac{a_{12} a_{16}-a_{11} a_{26}}{a_{12}^{2}-a_{11} a_{22}}$. Прямые поступательного воздействия, проходящие через точку $P_{1}$ и расположенные в плоскости $z=0$, приводят к поступательному перемещению в этой плоскости. Прямые поступательного воздействия, расположенные в плоскости $\left(y-y_{2}\right)\left(x-x_{3}\right)-\left(y-y_{1}\right)\left(x-x_{2}\right)=0$ и проходящие через точку $P_{2}$, приводят к поступательному движению в пространстве. Точки $P_{1}$ и $P_{2}$ находятся на прямой, по которой пересекаются эти две плоскости.

Ненулевые коэффициенты $a_{i j}$ приведены в табл. 3. При их вычислении принимаем $b=3,9 \mathrm{мм}, \quad a_{1}=\frac{b}{\sqrt{1-e_{t 1}^{2}}}, a_{2}=\frac{b}{\sqrt{1-e_{t 2}^{2}}}, e_{t 1}=0,6, e_{t 2}=0,2$. Остальные геометрические размеры корня зуба и постоянные упругости периодонтальной связки имеют прежние значения.

На рис. 2 показан корень зуба и плоскости, в которых расположены прямые поступательного воздействия (геометрические размеры корня зуба приведены выше, координаты точек $P_{1}$ и $P_{2}$ определены в соответствии с данными табл. 3).

Прямая $A B \quad$ (рис. 2, б) является линией пересечения двух плоскостей, содержащих прямые поступательного воздействия. Через точку $P_{1}$ в плоскости 1 проходят линии действия сил для поступательного перемещения в плоскости $x 0 y$, через точку $P_{2}$ в плоскости 2 проходят линии действия сил для поступательного перемещения в пространстве. Точки $P_{1}$ и $P_{2}$ принадлежат прямой $A B$ и имеют координаты $(0,234 ; 0 ; 8,600)$ и $(0,303 ; 0 ; 8,438)$ соответственно (значения координат приведены в миллиметрах). 
Коэффициенты $a_{i j}$ для модели корня зуба с одной плоскостью симметрии

\begin{tabular}{|c|c|c|c|c|c|}
\hline$a_{11}, \mathrm{MH} / \mathrm{M}$ & $a_{22}, \mathrm{MH} / \mathrm{M}$ & $a_{33}, \mathrm{MH} / \mathrm{M}$ & $a_{44}, \mathrm{H}$ & $a_{55}, \mathrm{H}$ & $a_{66}, \mathrm{H}$ \\
\hline 5,189 & 0,972 & 6,247 & 516,525 & 4,093 & 444,265 \\
\hline$a_{12}, \mathrm{KH} / \mathrm{M}$ & $a_{16}, \mathrm{\kappa H}$ & $a_{26}, \mathrm{\kappa H}$ & $a_{34}, \mathrm{\kappa H}$ & $a_{35}, \mathrm{\kappa H}$ & $a_{45}, \mathrm{H} \cdot \mathrm{M}$ \\
\hline$-92,470$ & $-44,639$ & 1,022 & 52,703 & $-1,890$ & $-17,526$ \\
\hline
\end{tabular}

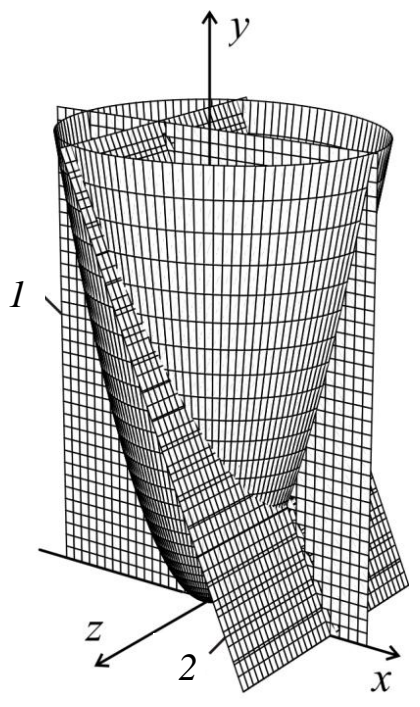

$a$

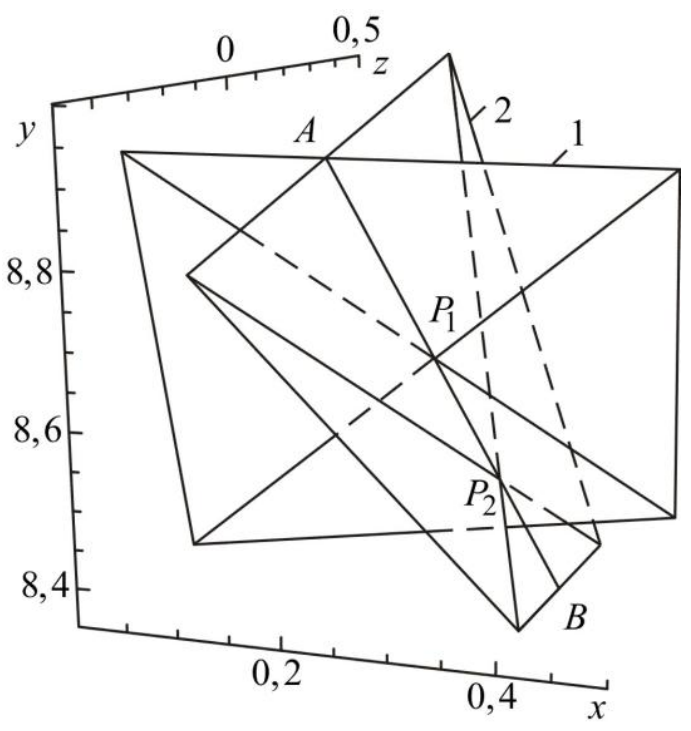

6

Рис. 2. Плоскости $1(z=0)$ и $\left(\left(y-y_{2}\right)\left(x-x_{3}\right)-\left(y-y_{1}\right)\left(x-x_{2}\right)=0\right)$, содержащие прямые поступательного воздействия $(a)$, для корня зуба с одной плоскостью симметрии и область сопротивления $P_{1}, P_{2}$ с прямыми поступательного воздействия (б)

\section{Модель корня зуба в форме асимметричного составного параболоида}

Если поверхность корня зуба описывается уравнениями четырех различных параболоидов, то все коэффициенты $a_{i j}, i, j=\overline{1,6}$ являются ненулевыми. Также отличны от нуля все компоненты матриц решения (5) и матриц $\delta$ и $\varepsilon$. Чтобы избежать громоздких выкладок, нахождение области сопротивления для асимметричной модели корня зуба выполним численно. Высота корня зуба $h=13,0$ мм, полуоси $b_{1}=3,9$ мм, $b_{2}=2,0 \mathrm{мM}, a_{1}=\frac{b_{1}}{\sqrt{1-e_{t 1}^{2}}}, a_{2}=\frac{b_{2}}{\sqrt{1-e_{t 2}^{2}}}, e_{t 1}=0,6, e_{t 2}=0,75$, постоянные упругости для периодонтальной ткани имеют прежние значения. Значения коэффициентов $a_{i j}$, $i, j=\overline{1,6}$ приведены в табл. 4 .

Собственные числа и векторы матрицы $\delta$ имеют вид

$$
\begin{gathered}
\delta_{1} \approx-261,80 \cdot 10^{-6}, \delta_{2} \approx 104,461 \cdot 10^{-6}, \delta_{3} \approx 153,038 \cdot 10^{-6}, \\
\vec{v}_{1} \approx(-0,630 ;-0,584 ; 0,514), \vec{v}_{2} \approx(0,717 ;-0,691 ; 0,093), \vec{v}_{3} \approx(0,301 ; 0,427 ; 0,853) .
\end{gathered}
$$


Отсюда следует, что асимметричный корень зуба принадлежит к типу $(+,+,-)$ по классификации [11]. Областью сопротивления в этом случае является горловой эллипс однополостного гиперболоида

$$
p_{11} x^{2}+p_{22} z^{2}+p_{33} y^{2}+p_{12} x z+p_{13} x y+p_{23} z y+p_{1} x+p_{2} z+p_{3} y+p_{0}=0 .
$$

Таблича 4

\section{Коэффициенты $a_{i j}$ в случае асимметричного корня зуба}

\begin{tabular}{|c|c|c|c|c|c|c|}
\hline$a_{11}, \mathrm{MH} / \mathrm{M}$ & $a_{22}, \mathrm{MH} / \mathrm{M}$ & $a_{33}, \mathrm{MH} / \mathrm{M}$ & $a_{12}, \mathrm{KH} / \mathrm{M}$ & $a_{23}, \mathrm{KH} / \mathrm{M}$ & $a_{13}, \mathrm{KH} / \mathrm{M}$ & $a_{34}, \mathrm{\kappa H}$ \\
\hline 3,849 & 0,629 & 5,832 & $-140,343$ & $-273,895$ & 97,168 & 47,783 \\
\hline$a_{44}, \mathrm{H} \cdot \mathrm{M}$ & $a_{55}, \mathrm{H} \cdot \mathrm{M}$ & $a_{66}, \mathrm{H} \cdot \mathrm{M}$ & $a_{45}, \mathrm{H} \cdot \mathrm{M}$ & $a_{46}, \mathrm{H} \cdot \mathrm{M}$ & $a_{56}, \mathrm{H} \cdot \mathrm{M}$ & $a_{35}, \mathrm{\kappa H}$ \\
\hline 458,902 & 9,017 & 319,459 & $-33,957$ & $-10,073$ & $-16,324$ & $-3,764$ \\
\hline$a_{14}, \mathrm{H}$ & $a_{15}, \mathrm{KH}$ & $a_{16}, \mathrm{\kappa H}$ & $a_{24}, \mathrm{KH}$ & $a_{25}, \mathrm{H}$ & $a_{26}, \mathrm{KH}$ & $a_{36}, \mathrm{H}$ \\
\hline 877,467 & 1,781 & $-32,500$ & $-2,346$ & 109,886 & 1,462 & $-987,353$ \\
\hline
\end{tabular}

Таблица 5

\section{Коэффициенты уравнения (7) однополостного гиперболоида}

\begin{tabular}{|c|c|c|c|c|c|c|c|c|c|}
\hline$p_{11}$ & $p_{22}$ & $p_{33}$ & $p_{12}$ & $p_{13}$ & $p_{23}$ & $p_{1}$ & $p_{2}$ & $p_{3}$ & $p_{0}$ \\
\hline$-2,117$ & 1,126 & 0,401 & 33,338 & $-43,455$ & 29,251 & 0,353 & $-0,262$ & 0,008 & $-0,000087$ \\
\hline
\end{tabular}

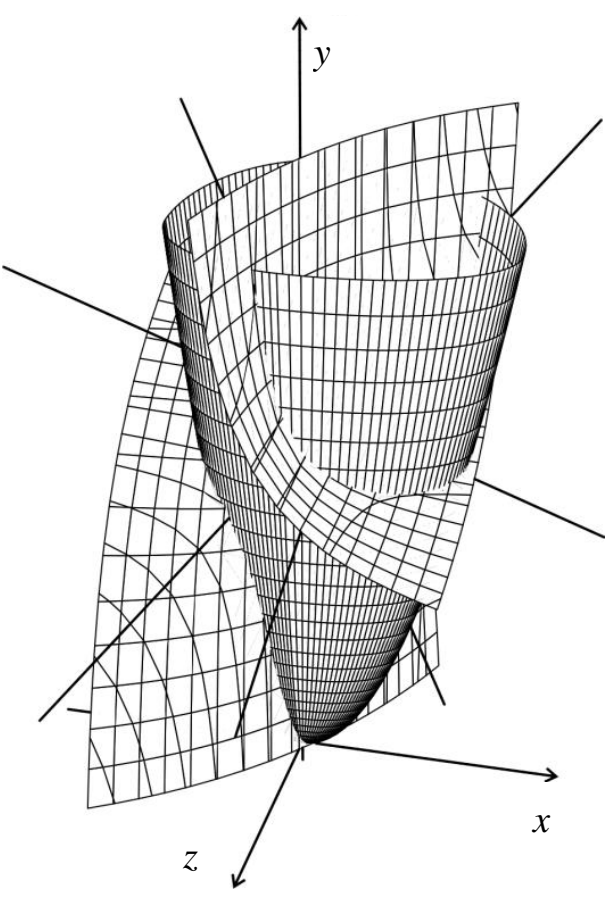

$a$

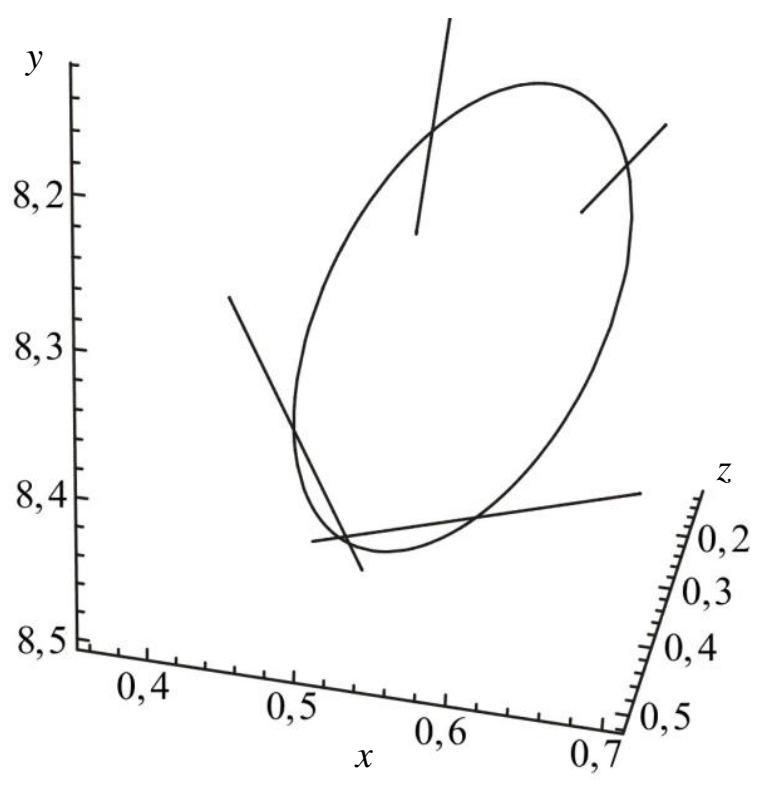

$\sigma$

Рис. 3. Прямые поступательного воздействия $(a)$, проходящие через горловой эллипс однополостного гиперболоида (8), и область сопротивления (б) для асимметричного корня зуба с прямыми поступательного воздействия (масштаб по координатным осям приведен в миллиметрах) 
Приближенные значения коэффициентов уравнения гиперболоида (7) приведены в табл. 5.

После приведения к каноническому виду уравнение (7) принимает вид

$$
\frac{z_{0}^{2}}{a_{z}^{2}}+\frac{y_{0}^{2}}{a_{y}^{2}}-\frac{x_{0}^{2}}{a_{x}^{2}}=1
$$

где $a_{x} \approx 0,126$ мкм, $a_{y} \approx 0,200$ мкм, $a_{z} \approx 0,165$ мкм. Координаты $\left(x_{0}, z_{0}, y_{0}\right)$ выражаются через координаты $(x, z, y)$ следующим образом:

$$
\begin{aligned}
& x_{0} \approx-0,005+0,629 x+0,584 y-0,514 z, \\
& y_{0} \approx-0,004+0,301 x+0,429 y+0,853 z, \\
& z_{0} \approx-0,005-0,717 x+0,691 y-0,093 z .
\end{aligned}
$$

Образующие однополостного гиперболоида (7), проходящие через точки горлового эллипса, являются прямыми поступательного воздействия $[6,11]$. На рис. 3 показаны корень зуба и область сопротивления (7), а также горловой эллипс с прямыми поступательного воздействия. Если линия действия нагрузки проходит вдоль прямой поступательного воздействия, зуб получает поступательное перемещение в пространстве.

Прямые поступательного воздействия, показанные на рис. $3, a$ и 6 , проходят через вершины горлового эллипса. Точки приложения нагрузок к корню зуба для его поступательного перемещения расположены на линиях пересечения однополостного гиперболоида и составного параболоида.

\section{ЗАКЛЮЧЕНИЕ}

1. Области сопротивления для симметричных и асимметричных корней зубов, внешняя поверхность которых может быть описана одним уравнением параболоида, а также двумя или четырьмя уравнениями различных параболоидов соответствуют типам областей сопротивлений, определенных в работах $[6,11]$.

2. Для корня зуба в форме параболоида с эллиптическим сечением, имеющим нулевой эксцентриситет, областью сопротивления является одна точка (единый центр сопротивления). Если эксцентриситет эллипса отличен от нуля, область сопротивления состоит из двух точек, расположенных на пересечении плоскостей симметрии, в которых лежат прямые поступательного воздействия. Расстояние между точками будет тем больше, чем больше эксцентриситет эллипса.

3. Для корня зуба, состоящего из двух параболоидов с полуэллиптическим сечением и одной общей полуосью, одна из плоскостей, содержащих прямые поступательного воздействия, является плоскостью симметрии. Вторая плоскость с прямыми поступательного воздействия перпендикулярна этой плоскости, но располагается не вертикально. Эта плоскость у альвеолярного гребня наклонена в сторону полуэллипса с меньшим эксцентриситетом.

4. Областью сопротивления асимметричного корня зуба, состоящего из четырех различных параболоидов, является горловой эллипс однополостного гиперболоида. Область сопротивления полностью расположена внутри корня. Прямолинейные образующие однополостного гиперболоида, проходящие через горловой эллипс, являются прямыми поступательного воздействия. 
5. Наибольшее расстояние между точками, ограничивающими область сопротивления для корня зуба в форме симметричного параболоида с эллиптическим сечением, составного параболоида с одной плоскостью симметрии и асимметричного составного параболоида, не превышает 0,3 мм. Для корней зубов указанной геометрической формы и корня зуба в форме кругового параболоида область сопротивления располагается на расстоянии, составляющем 64-65 \% от длины корня зуба (если считать от вершины корня). С учетом малых размеров области сопротивления и ее приближенной локализации наиболее важным для оценки условий поступательного перемещения корня зуба представляется расположение прямых поступательного воздействия и координат точки приложения нагрузки.

\section{БЛАГОДАРНОСТИ}

Работа выполнена при поддержке программы научных исследований FP7 IRSES Marie Curie (грант № 610547 TAMER).

\section{СПИСОК ЛИТЕРАТУРЫ}

1. Босяков С.М., Мселати А.Ф., Круподеров А.В. Математическое моделирование начальных перемещений корня зуба в форме двуполостного гиперболоида // Российский журнал биомеханики. 2015. - Т. 19, № 2. - С. 186-204.

2. Босяков С.М., Юркевич К.С. Определение жесткости костной ткани при поступательных перемещениях и поворотах корня зуба // Российский журнал биомеханики. - 2010. - Т. 14, № 2. C. $37-46$.

3. Bourauel C., Keilig L., Rahimi A., Reimann S., Ziegler A., Jager A. Computer-aided analysis of the biomechanics of tooth movements // Int. J. Comp. Dent. - 2007. - Vol. 10. - P. 25-40.

4. Cattaneo P. M., Dalstra M., Melsen B. Moment-to-force ratio, center of rotation, and force level: a finite element study predicting their interdependency for simulated orthodontic loading regimens // Am. J. Orthod. Dentofacial Orthop. - 2008. - Vol. 133. - P. 681-689.

5. Dathe H., Nägerl H., Kubein-Meesenburg D. A caveat concerning center of resistance // J. Dent. Biomech. 2013. - Vol. 4. - P. 1-7.

6. Dubinin A.L. Region of resistance of tooth: experimental determination // Russ. J. Biomech. - 2015. Vol. 19, No. 1. - P. 69-78.

7. Dubinin A.L., Nyashin Yu.I., Osipenko M.A. Analysis of development of the concept "center of resistance of tooth” // Russ. J. Biomech. - 2014. - Vol. 18, № 4. - P. 393-409.

8. Halazonetis D.J. Ideal arch force systems: a center-of-resistance perspective // Am. J. Orthod. Dentofacial Orthop. - 1998. - Vol. 114. - P. 256-264.

9. Hohmann A., Kober C., Young P., Dorow C., Geiger M., Andrew Boryor A., Sander F. M., Sander C., Sander F.G. Influence of different modeling strategies for the periodontal ligament on finite element simulation results // Am. J. Orthod. Dentofacial Orthop. - 2011. - Vol. 139. - P. 775-783.

10. Meyer B.N., Chen J., Katona T.R. Does the center of resistance depend on the direction of tooth movement? // Am. J. Orthod. Dentofacial Orthop. - 2010. - Vol. 137. - P. 354-361.

11. Osipenko M.A., Nyashin Yu.I., Nyashin M.Yu., Dubinin A.L. Region of resistance of a tooth: the definition and the properties // Russ. J. Biomech. - 2013. - Vol. 17, № 2. - P. 25-31.

12. Provatidis C.G. An analytical model for stress analysis of a tooth in translation // Int. J. Eng. Sci. - 2001. Vol. 39. - P. 1361-1381.

13. Rees J.S., Jacobsen P.H. Elastic modulus of the periodontal ligament // Biomat. - 1997. - Vol. 18. P. 995-999.

14. Smith R.J., Burstone C.J. Mechanics of tooth movement // Am. J. Orthod. - 1984. - Vol. 85. - P. $294-307$.

15. Tanne K., Nagataki T., Inoue Y., Sakuda M., Burstone C.J. Patterns of initial tooth displacements associated with various root lengths and alveolar bone heights // Am. J. Orthod. Dentofacial Orthop. - 1991. Vol. 100. - P. 66-71.

16. Van Schepdael A., Geris L., Van der Sloten J. Analytical determination of stress patterns in the periodontal ligament during orthodontic tooth movement // Med. Eng. Phys. - 2013. - Vol. 35. - P. 403-410.

17. Viecilli R.F. Self-corrective T-loop design for differential space closure // Am. J. Orthod. Dentofacial Orthop. - 2006. - Vol. 129. - P. 48-53. 
18. Viecilli R.F., Budiman A., Burstone C.J. Axes of resistance for tooth movement: Does the center of resistance exist in 3-dimensional space? // Am. J. Orthod. Dentofacial Orthop. - 2013. - Vol. 143. P. 163-172.

19. Viecilli R.F., Katona T.R., Chen J., Hartsfield J.K.Jr., Roberts W.E. Orthodontic mechanotransduction and the role of the P2X7 receptor // Am. J. Orthod. Dentofacial Orthop. - 2009. - Vol. 135. P. 694.e1-694.e16.

20. Viecilli R.F., Katona T.R., Chen J., Hartsfield J.K.Jr., Roberts W.E. Three-dimensional mechanical environment of orthodontic tooth movement and root resorption // Am. J. Orthod. Dentofacial Orthop. 2008. - Vol. 133. - P. 711-726.

21. Vollmer D., Bourauel C., Maier K., Jäger A. Determination of the centre of resistance in an upper human canine and idealized tooth model // Eur. J. Orthod. - 1999. - Vol. 21. - P.633-648.

\section{ПРИЛОЖЕНИЕ}

Выражения для коэффициентов $a_{i j}(E, v-$ модуль упругости и коэффициент Пуассона периодонтальной связки):

$$
\begin{aligned}
& a_{11}=A \sum_{k=1}^{4} \iint_{F_{k}}\left(n_{y}^{(k)}(1-2 v) \cos \left(\alpha_{k}\right)-\left(2 H_{k} n_{x}^{(k)}(1-v)+G_{k} n_{z}^{(k)}(1-2 v)\right) \sin \left(\alpha_{k}\right)\right) d F_{k}, \\
& a_{12}=a_{21}=A(1-2 v) \sum_{k=1}^{4} \iint_{F_{k}}\left(2 v n_{x}^{(k)} \cos \left(\alpha_{k}\right)-H_{k} n_{y}^{(k)}(1-2 v) \sin \left(\alpha_{k}\right)\right) d F_{k}, \\
& a_{13}=a_{31}=-A \sum_{k=1}^{4} \iint_{F_{k}}\left(2 G_{k} v n_{x}^{(k)}+H_{k}(1-2 v) n_{z}^{(k)}\right) \sin \left(\alpha_{k}\right) d F_{k}, \\
& a_{14}=a_{41}=-A \sum_{k=1}^{4} \iint_{F_{k}}\left(2 v n_{x}^{(k)} z \cos \left(\alpha_{k}\right)+\left(2 G_{k}^{3} v n_{x}^{(k)} y+2 G_{k} H_{k}^{2} v n_{x}^{(k)} y+\right.\right. \\
& \left.\left.+H_{k}(1-2 v)\left(H_{k}^{2} n_{z}^{(k)} y-n_{y}^{(k)} z\right)\right) \sin \left(\alpha_{k}\right)\right) d F_{k}, \\
& a_{15}=a_{51}=-A \sum_{k=1}^{4} \iint_{F_{k}}\left((1-2 v) n_{y}^{(k)} z \cos \left(\alpha_{k}\right)+\left(H_{k}\left(n_{z}^{(k)} x(1-2 v)-2 z n_{x}^{(k)}(1-v)\right)+\right.\right. \\
& \left.\left.+G_{k}\left(2 v\left(x n_{x}^{(k)}+z n_{z}^{(k)}\right)-z n_{z}^{(k)}\right)\right) \sin \left(\alpha_{k}\right)\right) d F_{k}, \\
& a_{16}=a_{61}=A \sum_{k=1}^{4} \iint_{F_{k}}\left(\left(y n_{y}^{(k)}-2 v\left(x n_{x}^{(k)}+y n_{y}^{(k)}\right)\right) \cos \left(\alpha_{k}\right)-\right. \\
& -\left(2 H_{k}^{3} y n_{x}^{(k)}(1-v)+G_{k}^{3} y n_{z}^{(k)}(1-2 v)+G_{k} H_{k}^{2} y n_{z}^{(k)}(1-2 v)-\right. \\
& \left.\left.-H_{k}\left(x n_{y}^{(k)}(1-2 v)-2 G_{k}^{2} y n_{x}^{(k)}(1-v)\right)\right) \sin \left(\alpha_{k}\right)\right) d F_{k}, \\
& a_{22}=A \sum_{k=1}^{4} \iint_{F}\left(2 n_{y}^{(k)}(1-v) \cos \left(\alpha_{k}\right)-(1-2 v)\left(H_{k} n_{x}^{(k)}+G_{k} n_{z}^{(k)}\right) \sin \left(\alpha_{k}\right)\right) d F_{k} \text {, } \\
& a_{23}=a_{32}=A \sum_{k=1}^{4} \iint_{F_{k}}\left(n_{z}^{(k)}(1-2 v) \cos \left(\alpha_{k}\right)-2 G_{k} v n_{y}^{(k)} \sin \left(\alpha_{k}\right)\right) d F_{k},
\end{aligned}
$$




$$
\begin{aligned}
& a_{24}=a_{42}=A \sum_{k=1}^{4} \iint_{F}\left(\left(y n_{z}^{(k)}(1-2 v)-2 z n_{y}^{(k)}(1-v)\right) \cos \left(\alpha_{k}\right)+\right. \\
& +\left(H_{k} z n_{x}^{(k)}(1-2 v)-2 G_{k}^{3} y n_{y}^{(k)} v-G_{k}\left(2 H_{k}^{2} y n_{y}^{(k)} v-z n_{z}^{(k)}(1-2 v)\right) \sin \left(\alpha_{k}\right)\right) d F_{k}, \\
& a_{25}=a_{52}=A \sum_{k=1}^{4} \iint_{F}\left(\left(z n_{x}^{(k)}-x n_{z}^{(k)}\right)(1-2 v) \cos \left(\alpha_{k}\right)+2 n_{y}^{(k)} v\left(G_{k} x-H_{k} z\right) \sin \left(\alpha_{k}\right)\right) d F_{k}, \\
& a_{26}=a_{62}=A \sum_{k=1}^{4} \iint_{F}\left(\left(2 x n_{y}^{(k)}(1-v)-y n_{x}^{(k)}(1-2 v)\right) \cos \left(\alpha_{k}\right)-\right. \\
& \left.-\left(G_{k} x n_{z}^{(k)}(1-2 v)-2 H_{k}^{3} y n_{y}^{(k)} v+H_{k}\left(x n_{x}^{(k)}(1-2 v)-2 G_{k}^{2} y n_{y}^{(k)} v\right)\right) \sin \left(\alpha_{k}\right)\right) d F_{k}, \\
& a_{33}=A \sum_{k=1}^{4} \iint_{F_{k}}\left(n_{y}^{(k)}(1-2 v) \cos \left(\alpha_{k}\right)-\left(H_{k} n_{x}^{(k)}(1-2 v)+2 G_{k} n_{z}^{(k)}(1-v)\right) \sin \left(\alpha_{k}\right)\right) d F_{k}, \\
& a_{34}=a_{43}=A \sum_{k=1}^{4} \iint_{F_{k}}\left(\left(y n_{y}^{(k)}(1-2 v)-2 z n_{z}^{(k)} v\right) \cos \left(\alpha_{k}\right)-\right. \\
& \left.-\left(H_{k} y n_{x}^{(k)}(1-2 v)+2 G_{k}^{3} y n_{z}^{(k)}(1-v)+G_{k}\left(2 H_{k}^{2} y n_{z}^{(k)}(1-v)-z n_{y}^{(k)}(1-2 v)\right)\right) \sin \left(\alpha_{k}\right)\right) d F_{k} \text {, } \\
& a_{35}=a_{53}=-A \sum_{k=1}^{4} \iint_{F_{k}}\left(x n_{y}^{(k)}(1-2 v) \cos \left(\alpha_{k}\right)+\left(G_{k}\left(2 x n_{z}^{(k)}(1-v)-z n_{x}^{(k)}(1-2 v)\right)+\right.\right. \\
& \left.\left.+H_{k}\left(x n_{x}^{(k)}(1-2 v)-2 z n_{z}^{(k)} v\right)\right) \sin \left(\alpha_{k}\right)\right) d F_{k}, \\
& a_{36}=a_{63}=A \sum_{k=1}^{4} \iint_{F_{k}}\left(2 x n_{z}^{(k)} v \cos \left(\alpha_{k}\right)+\left(G_{k}^{3} y n_{x}^{(k)}(1-2 v)+2 H_{k} y n_{z}^{(k)} v-\right.\right. \\
& \left.\left.-G_{k}(1-2 v)\left(x n_{y}^{(k)}-H_{k}^{2} y n_{x}^{(k)}\right)\right) \sin \left(\alpha_{k}\right)\right) d F_{k}, \\
& a_{44}=-A \sum_{k=1}^{4} \iint_{F_{k}}\left(\left((1-2 v)\left(z n_{z}^{(k)}-y n_{y}^{(k)}\right) y+2 z\left(v y n_{z}^{(k)}-z n_{y}^{(k)}(1-v)\right)\right) \cos \left(\alpha_{k}\right)+\right. \\
& +\left(2 G_{k}^{3} y\left((1-v) y n_{z}^{(k)}-v z n_{y}^{(k)}\right)+H_{k} G_{k}^{2} y^{2} n_{x}^{(k)}+\right. \\
& +G_{k}\left((1-2 v) z\left(z n_{z}^{(k)}-y n_{y}^{(k)}\right)+2 H_{k}^{2} y\left((1-v) y n_{z}^{(k)}-v z n_{y}^{(k)}\right)\right)+ \\
& \left.\left.+H_{k}(1-2 v) n_{x}^{(k)}\left(H_{k}^{2} y^{2}+z^{2}\right)\right) \sin \left(\alpha_{k}\right)\right) d F_{k}, \\
& a_{45}=c_{54}=-A \sum_{k=1}^{4} \iint_{F_{k}}\left((1-2 v)\left(x y n_{y}^{(k)}+z\left(z n_{x}^{(k)}-x n_{z}^{(k)}\right)\right) \cos \left(\alpha_{k}\right)+\right. \\
& +\left(G_{k}\left(z\left(2 v x n_{y}^{(k)}+y n_{x}^{(k)}-2 v y n_{x}^{(k)}\right)-2(1-v) x y n_{z}^{(k)}\right)+\right. \\
& \left.\left.+H_{k}\left(2 v z\left(y n_{z}^{(k)}-z n_{y}^{(k)}\right)-(1-2 v) x y n_{x}^{(k)}\right)\right) \sin \left(\alpha_{k}\right)\right) d F_{k},
\end{aligned}
$$




$$
\begin{aligned}
& a_{46}=a_{64}=A \sum_{k=1}^{4} \iint_{F_{k}}\left(\left(z\left(y n_{x}^{(k)}-2 x n_{y}^{(k)}\right)+2 v\left(z\left(x n_{y}^{(k)}-y n_{x}^{(k)}\right)+x y n_{z}^{(k)}\right)\right) \cos \left(\alpha_{k}\right)+\right. \\
& +\left(G_{k}^{3}(1-2 v) y^{2} n_{x}^{(k)}+2 G_{k}^{2} H_{k} v y\left(y n_{z}^{(k)}-z n_{y}^{(k)}\right)-G_{k}(1-2 v)\left(x\left(y n_{y}^{(k)}-z n_{z}^{(k)}\right)-H_{k}^{2} y^{2} n_{x}^{(k)}\right)+\right. \\
& \left.\left.+H_{k}\left((1-2 v) x z n_{x}^{(k)}+2 H_{k}^{2} v y\left(y n_{z}^{(k)}-z n_{y}^{(k)}\right)\right)\right) \sin \left(\alpha_{k}\right)\right) d F_{k}, \\
& a_{55}=A \sum_{k=1}^{4} \iint_{F_{k}}\left((1-2 v)\left(x^{2}+z^{2}\right) n_{y}^{(k)} \cos \left(\alpha_{k}\right)+\right. \\
& +\left(H_{k}\left(x z n_{z}^{(k)}-\left((1-2 v) x^{2}+2(1-v) z^{2}\right) n_{x}^{(k)}\right)+\right. \\
& \left.+G_{k}\left(x z n_{x}^{(k)}-\left((1-2 v) x^{2}+2(1-v) z^{2}\right) n_{z}^{(k)}\right)\right) \sin \left(\alpha_{k}\right) d F_{k}, \\
& a_{56}=a_{65}=-A \sum_{k=1}^{4} \iint_{F_{k}}\left(y z n_{y}^{(k)}+2 v\left(x^{2} n_{z}^{(k)}-\left(x n_{x}^{(k)}+y n_{y}^{(k)}\right) z\right) \cos \left(\alpha_{k}\right)+\right. \\
& +\left(2 G_{k}^{2} H_{k} y\left(v\left(x n_{z}^{(k)}+z n_{x}^{(k)}\right)-z n_{x}^{(k)}\right)+G_{k}^{3}(1-2 v) y\left(x n_{x}^{(k)}-z n_{z}^{(k)}\right)+\right. \\
& +H_{k}\left((1-2 v) x z n_{y}^{(k)}+2 H_{k}^{2} y\left(v\left(x n_{z}^{(k)}+z n_{x}^{(k)}\right)-z n_{x}^{(k)}\right)\right)- \\
& \left.\left.-G_{k}(1-2 v)\left(x^{2} n_{y}^{(k)}+H_{k}^{2} y\left(z n_{z}^{(k)}-x n_{x}^{(k)}\right)\right)\right) \sin \left(\alpha_{k}\right)\right) d F_{k} \\
& a_{66}=-A \sum_{k=1}^{4} \iint_{F_{k}}\left(\left(x y n_{x}^{(k)}-n_{y}^{(k)}\left(2(1-v) x^{2}+(1-2 v) y^{2}\right)\right) \cos \left(\alpha_{k}\right)+\right. \\
& +\left(G_{k} H_{k}^{2}(1-2 v) n_{z}^{(k)} y^{2}-2 H_{k}^{3} y\left(v x n_{y}^{(k)}-(1-v) y n_{x}^{(k)}\right)+G_{k}(1-2 v)\left(x^{2}+G_{k}^{2} y^{2}\right)+\right. \\
& \left.\left.+H_{k}\left(\left(2 v H_{k}^{2}-1\right) x y n_{y}^{(k)}+n_{x}^{(k)}\left((1-2 v) x^{2}+2 G_{k}^{2}(1-v) y^{2}\right)\right)\right) \sin \left(\alpha_{k}\right)\right) d F_{k}, \\
& A=\frac{E}{2 \delta(1+v)(1-2 v)}, H_{k}=\frac{x\left(1-e_{k}^{2}\right)}{\sqrt{x^{2}\left(1-e_{k}^{2}\right)^{2}+z^{2}}}, G_{k}=\frac{z}{\sqrt{x^{2}\left(1-e_{k}^{2}\right)^{2}+z^{2}}}, \\
& e_{1}=e_{t 1}, e_{2}=\frac{1}{b_{2}} \sqrt{b_{1}^{2}\left(1-e_{t 2}^{2}\right)+b_{2}^{2}}, e_{3}=e_{t 2}, e_{4}=\frac{1}{b_{1}} \sqrt{b_{1}^{2}+b_{2}^{2}\left(1-e_{t 1}^{2}\right)} \text {, } \\
& \operatorname{tg}\left(\alpha_{1}\right)=\frac{2 h \sqrt{x^{2}\left(1-e_{t 1}^{2}\right)^{2}+z^{2}}}{b_{1}^{2}}, \operatorname{tg}\left(\alpha_{2}\right)=2 h \sqrt{\frac{x^{2}\left(1-e_{t 2}^{2}\right)^{2}}{b_{2}^{4}}+\frac{z^{2}}{b_{1}^{4}}}, \\
& \operatorname{tg}\left(\alpha_{3}\right)=\frac{2 h \sqrt{x^{2}\left(1-e_{t 2}^{2}\right)^{2}+z^{2}}}{b_{2}^{2}}, \operatorname{tg}\left(\alpha_{4}\right)=2 h \sqrt{\frac{x^{2}\left(1-e_{t 1}^{2}\right)^{2}}{b_{1}^{4}}+\frac{z^{2}}{b_{2}^{4}}} \text {. }
\end{aligned}
$$




\section{REGIONS OF RESISTANCE FOR MODEL OF THE SINGLE-ROOTED TOOTH: DIFFERENT SYMMETRY CASES}

\section{S.M. Bosiakov, A.F. Mselati (Minsk, Belarus)}

Regions of resistance and the positions of lines of the translational action for the model of the tooth root in the form of a complex paraboloid based on approach suggested by A.L. Dubinin, Yu.I. Nyashin, and M.A. Osipenko were defined. The stiffness of the periodontal ligament is obtained by solving the problem of equilibrium of the elastically fixed rigid body under the action of a concentrated force. The periodontal ligament is linearly elastic and nearly incompressible material; its thickness is constant along the normal to the external surface. The small movement of the tooth root was expressed with a combination of translational displacement and rotational angle. The total strain of the periodontal tissue normal to the surface of the root was assessed in conjunction with the width of the periodontal ligament in this direction. External surface of the tooth root and periodontal ligament was described by means of equations of paraboloids. The equations of the resistance area for an asymmetric tooth root, tooth root with a single plane of symmetry, and a symmetrical tooth root with an elliptical and circular cross-section are derived. It has been indicated that the resistance region for the asymmetric tooth root is gorge ellipse of the one-sheeted hyperboloid; the resistance region for the tooth root with a single plane of symmetry and the symmetric tooth root with elliptical cross-section are two points; a single centre of resistance is occurred for symmetric tooth root with a circular cross-section. The calculations of the resistance region locations for asymmetrical and symmetrical teeth roots with different geometric dimensions were carried out. The resistance regions, as well as lines of translational action for the tooth root with a single plane of symmetry and asymmetric tooth root were plotted. The obtained results can be used to predict the regions of the load application and direction of the loading for the translational displacements of the tooth root.

Key words: region of resistance, asymmetrical tooth root, periodontal ligament, paraboloid, line of translational action. 